\title{
Polymer MS-MS by MALDI: some advances and some challenges
}

\author{
David M. Hercules
}

Published online: 4 August 2008

(C) Springer-Verlag 2008

MALDI mass spectrometry has been making a significant impact of the analysis of synthetic polymers. Evidence is its citation by major chapters in monographs on polymer mass spectrometry $[1,2]$, and a separate monograph on MALDI of synthetic polymers [3]. The purpose of this special issue is to illustrate results from the next step in the use of MALDI for polymer characterization.

What has MALDI accomplished to date? The following list indicates analyses for which conventional MALDI is useful, but the list is not exhaustive:

1. identification - the polymer repeat unit mass;

2. end groups - exact mass measurements give information about end groups;

3. minor species-MALDI is particularly useful for cyclic oligomers;

4. molecular weight distributions - particularly good for low-mass polymers;

5. branching - can detect branching in a variety of polymers;

6. size-exclusion chromatography — used as an absolute calibrant; and

7. copolymers - can distinguish between random, ordered, and block copolymers.

Despite the successes enumerated above, conventional MALDI often fails for specific types of polymer analysis. This issue will show the potential for overcoming some of these shortcomings by use of MALDI MS-MS. MALDI MS-MS is valuable for studying polymer decomposition

D. M. Hercules $(\bowtie)$

Department of Chemistry, Vanderbilt University,

Nashville, TN 37235, USA

e-mail: david.m.hercules@vanderbilt.edu mechanisms and the ability to correlate these mechanisms with pyrolysis GC-MS. These involve the same initial polymer chain fragmentation reactions, and the unimolecular decomposition reactions studied by MALDI help to explain pyrolysis GC-MS results in the condensed phase. Below I will discuss the important examples of MS-MS for polymer analysis - end group and repeat unit measurement.

End-group analysis by MALDI is best discussed with a specific example. In a spectrum of a poly $(\alpha$-methylstyrene $)$ one selects an intense oligomer peak for end-group analysis. The measured mass is $1417.8 \mathrm{Da}$; it is Ag-cationized. Subtracting the mass of $\mathrm{Ag}$ gives an oligomer mass of 1310.9 Da. Dividing this by the repeat unit mass of poly $(\alpha-$ methylstyrene) (118.08) gives 11.1 for a combined endgroup mass of $12.01 \mathrm{Da}$. This is clearly too small so it must be a 10-mer which, by the same process gives a combined end-group mass of $130.1 \mathrm{Da}$. Unless one has independent information about the polymer, this is not terribly helpful. However, MS-MS of the polymer gives two easily identifiable series of peaks each of which contains one of the terminal groups. Analyses of these series reveal the masses of the two end groups: $57.0 \mathrm{Da}$ and $73.1 \mathrm{Da}$. The former is probably a sec-butyl group which is a common polymer initiator. The latter most likely corresponds to $\mathrm{C}_{4} \mathrm{H}_{9} \mathrm{O}$ which comes from an epoxide termination reaction. To make positive identifications, however, an additional stage of MS-MS would be required to determine the exact endgroup structures.

Polymer repeat unit analysis also can contain vagaries. Consider a measured repeat unit mass of 118.08. Hypothetically, this could be one of five polymers: $\operatorname{poly}(\alpha-$ methylstyrene), poly( $\beta$-methylstyrene), poly(2-methylstyrene), poly(3-methylstyrene), or poly(4-methylstyrene). How well can MALDI MS-MS help us here? Again this is best seen by an example. In reality, it is probably a choice between the 

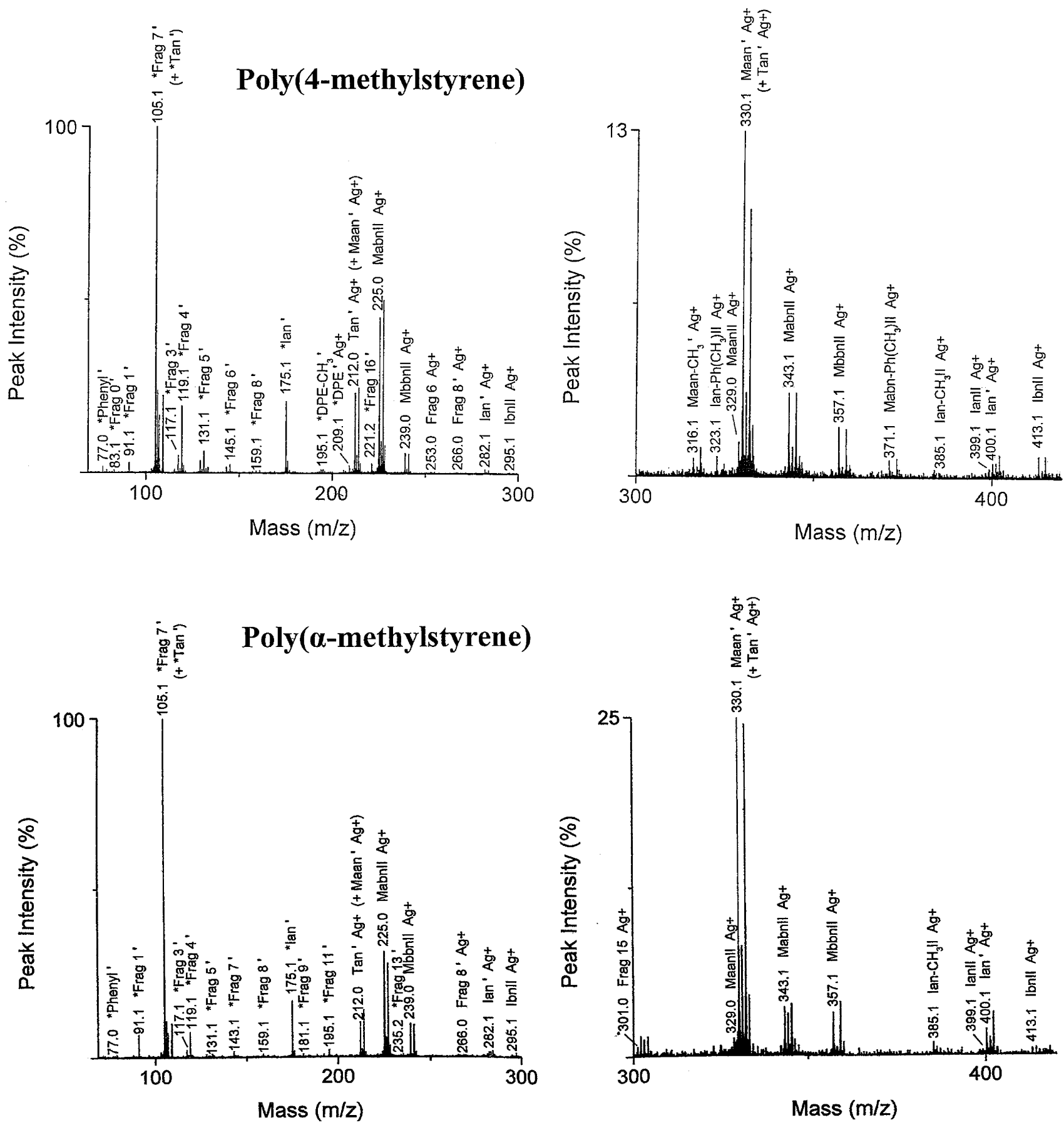

Fig. 1 MALDI MS-MS of poly(4-methylstyrene) and poly( $\alpha$-methylstyrene)

poly $\alpha$-methyl and poly 4-methyl polymers, the two most common examples. Figure 1 shows segments of the MALDI MS-MS spectra of these two polymers. The similarity between the two is striking! A weak peak pair at 371.1 and 371.3 Da occurs in the poly 4-methyl polymer but not the poly $\alpha$-methyl derivative, a significant but hardly compelling difference!. Again, an additional stage of MS-MS would answer the question definitively.
In summary, then, MALDI MS-MS offers a new and different dimension to polymer analysis. The first-order measurements are not necessarily always going to be definitive, but by using two stages of polymer MS-MS, one should have a very beneficial new approach to polymer characterization.

My special thanks the authors for submitting their contributions and to the reviewers for their expert comments. 


\section{References}

1. Montaudo G, Lattimer RP (eds) (2002) Mass spectrometry of polymers. CRC Press, Boca Raton, FL

2. Hillenkamp F, Peter-Katalini J (eds) (2007) MALDI MS. A practical guide to instrumentation, methods and applications. Wiley-VCH, Weinheim

3. Pasch H, Schrepp W (2003) MALDI-TOF mass spectrometry of synthetic polymers. Springer, Berlin Heidelberg New York

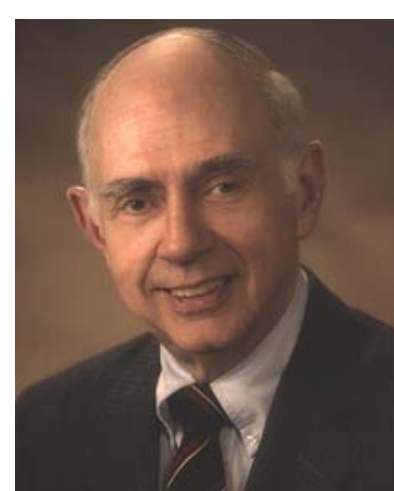

David M. Hercules is Centennial Professor of Chemistry, Emeritus at Vanderbilt University. His current research is on mass spectrometry of synthetic polymers, primarily using MALDI as the technique of choice. Special emphasis is on the use of MS-MS for analytical applications and to study polymer decomposition mechanisms. A second major emphasis is on MALDI of insoluble polymers. He is also investigating the use of IMS/MALDI for polymer analysis. 